\title{
Débitmétrie en hydrogène liquide
}

\author{
A. Memponteil \\ C.E.A./C.E.N.G. \\ Direction des réacteurs nucléaires \\ Département de thermohydraulique et de physique \\ S.T.I./L.A.S.P.
}

Les travaux sur la débitmétrie LH2 présentés ici sont destinés à l'instrumentation d'un banc d'étude des écoulements hydrogène liquide qui a été réalisé au CENG par le Département de Thermohydraulique et de Physique.

Le D.T.P. développe également un code de calcul (SIDHONY) modélisant les écoulements cryogéniques diphasiques qui interviennent lors de l'allumage des moteurs cryotechniques du lanceur européen ARIANE.

Le banc cryotechnique est destiné aux études expérimentales en hydrogène liquide, venant en support à l'élaboration du code de calcul.

Ces travaux sont réalisés dans le cadre du Programme de Recherche et Technologie du C.N.E.S. et de la S.E.P. et font l'objet d'une convention entre le C.E.A., le C.N.E.S. et la S.E.P.

\section{Description de l'installation}

L'installation d'essais figure 1, baptisée Mhynos, permet des études analytiques ou technologiques sur des écoulements d'hydrogène liquide, diphasique ou supercritique.

Les mesures de caractérisations d'écoulement sont réalisées au cours de rafales pendant lesquelles un réservoir de 300 litres est vidangé à travers une section d'essais instrumentée.

Le cycle expérimental commence par la mise en froid de l'installation jusqu'à $90 \mathrm{~K}$ avec de l'azote liquide, l'ensemble des circuits est ensuite pompé à $10^{-3}$ mbar avant mise en hydrogène. La mise en froid est ensuite achevée avec de l'hydrogène liquide avant remplissage du volume de 300 litres et des circuits.

Les structures et le fluide sont ensuite portés à la température prévue pour les essais par réchauffage, enfin l'installation est pressurisée.

Pendant la rafale, le réservoir est maintenu à pression constante, les conditions thermohydrauliques d'entrée dans la section d'essais sont contrôlées par une vanne de réglage.
Les mesures caractérisant l'écoulement dans la section d'essais sont enregistrées lorsque les critères de stabilité de l'écoulement sont satisfaits pour les essais stationnaires ou pendant une rampe imposée sur un paramètre.

Le réglage de vanne est ensuite modifié afin de changer les conditions d'entrée dans la section d'essais et l'enregistrement suivant intervient après une nouvelle stabilisation.

Au cours d'une rafale le nombre d'enregistrements dépend du débit d'hydrogène et du temps de stabilisation de l'écoulement.

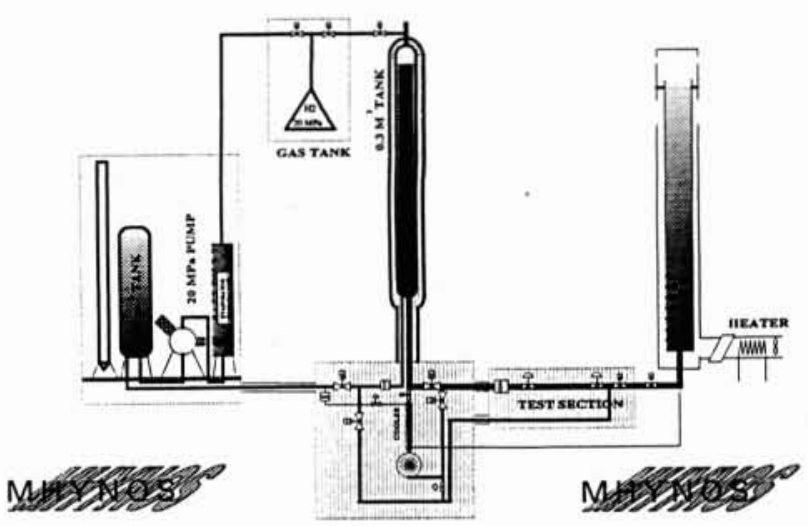

1. Installation d'essais Mhynos.

\section{Objet des études}

Le programme expérimental de l'installation vise la constitution d'une banque de données permettant dans un premier temps de valider les modèles physiques des différentes lois de fermetures du système d'équations du code

\section{Flowmetry in liquid hydrogen}

Two types of flowmeters were seen as possible for the characterization of the flow in liquid hydrogen and supercritical fluids : Coriolis and turbine flowmeters. The first one is a standard flowmeter. The second one is a prototype fitted to the use. The authors have completed validation measures processes. They present the first issues. 
Sidonhy et dans un deuxième temps de qualifier le code. Les deux objectifs imposent pour l'instrumentation des contraintes différentes.

La validation des modèles nécessite principalement une précision de l'ordre du pour-cent, sans contrainte de temps de réponse dans la mesure ou celui-ci ne ralentit pas le déroulement de la rafale. Les essais de qualification donnent lieu à des écoulements transitoires qui peuvent être rapides, ils nécessitent de la part de l'instrumentation un temps de réponse plus court.

\section{Spécification de l'instrumentation de débits}

La gamme de paramètres couverte par l'installation et dans laquelle les mesures de débits doivent être opérationnelles est relativement large, en premier lieu par la gamme de débits, en deuxième lieu par les caractéristiques physiques du fluide.

Les pré-calculs des essais planifiés sur l'installation nous ont amenés à prévoir une plage de débits allant de 2 à $200 \mathrm{~g} / \mathrm{s}$ ce qui représente une rangeabilité importante.

Le fluide, dont il faut mesurer le débit, peut être un liquide largement sous-refroidi, un fluide supercritique dont le comportement est proche d'un gaz, en passant par un liquide proche de la saturation. Dans tous les cas le fluide reste toutefois monophasique. La gamme de paramètres inclus la zone entourant le point critique dans laquelle le comportement du fluide est mal connu et les tables peu précises.

La pression va de 2 à 100 bar pour une température de 23 à $75 \mathrm{~K}$, sur cette plage de paramètres la densité passe de 35 à $70 \mathrm{~kg} / \mathrm{m}^{3}$.

La bande passante souhaitable des débitmètres pour les essais de qualification est de $50 \mathrm{~Hz}$ pour caractériser des écoulements oscillants dont la période est estimée à $5 \mathrm{~Hz}$. Actuellement cet objectif n'est pas une priorité et les efforts sont portés vers l'objectif de précision.

\section{Problèmes en débitmétrie hydrogène liquide}

\subsection{Problèmes liés à la cryogénie}

Les problèmes liés à l'aspect cryogénique d'une installation d'essais en hydrogène liquide sont dus, essentiellement, à la phase de refroidissement puis au comportement du matériel à basse température.

La mise en froid d'une installation cryogénique donne lieu à des écoulements dont il est difficile de maîtriser le débit volumique et la composition en phase vue par les débitmètres.

Dans la première phase de refroidissement, la vaporisation du fluide au contact des structures chaudes est très rapide et génère du gaz qui se réchauffe par convection, le tout provoquant une augmentation considérable du débit volumique.

En fin de mise en froid, l'écoulement présente un caractère diphasique avec une structure d'écoulement qui peut solliciter sévèrement les débitmètres.

Enfin la variation rapide de température du débitmètre est un facteur de détérioration à prendre en compte.

La conception de notre installation permet de détourner la plus grande partie des gaz générés pendant la mise en froid hors de la conduite d'essais, ce qui minimise les risques de sur-débits et de dégradation par des écoulements diphasiques, sans ralentir la mise en froid.

La vitesse de mise en froid est par ailleurs contrôlée et le refroidissement se fait principalement par convection de gaz plutôt que par la progression d'un front de trempe.

Le choix du débitmètre doit prendre en compte le comportement des matériaux qui le constituent à basse température en particulier la fragilisation, les dilatations différentielles, les changements de résistivité électrique, etc...

Enfin dans notre cas les débitmètres sont dans des doubles enceintes sous vide ce qui peut générer des contraintes supplémentaires.

\subsection{Problèmes spécifiques à l'hydrogène liquide}

Ils sont dus en premier lieu aux caractéristiques physiques de l'hydrogène liquide qui sont peu favorables aux turbines.

La faible densité $\left(70 \mathrm{~kg} / \mathrm{m}^{3}\right)$ est pénalisante pour la dynamique des turbines.

La faible viscosité (1E-5 Ns/ $\mathrm{m}^{2}$ ) impose pour ces mêmes turbines des montages sur roulements spéciaux autolubrifiés.

Le caractère dangereux de l'hydrogène crée une obligation d'étanchéité aux connections et astreint à se mettre en conformité avec la réglementation concernant les atmosphères explosibles.

Enfin l'incompatibilité de certains matériaux avec l'hydrogène restreint encore le champ des possibilités.

\section{Choix de la technologie de mesure}

Parmi les technologies classiques de débitmétrie, certaines ont été éliminées après une première analyse essentiellement pour deux motifs.

D'une part l'absence de matériels existant pour les applications en hydrogène liquide pour des raisons d'incompatibilités avec l'hydrogène ou les conditions d'utilisations.

D'autre part certaines technologies sont basées sur des principes physiques mettant en jeu des caractéristiques du fluide qui dans notre cas présentent une très grande variabilité, ce qui rend ces matériels très difficiles à étalonner pour l'ensemble de notre gamme d'utilisation.

Les débitmètres à ultrasons et électromagnétiques font partie de la première catégorie.

Les débitmètres à vortex sont inclus dans les deux catégories.

Les débitmètres déprimogènes ont donné lieu à une étude plus approfondie bien qu'ils puissent être classés dans la deuxième catégorie.

En effet cette technique de mesure est d'un usage très classique en cryogénie (hélium liquide par exemple). Les avantages en sont évidents, elle ne présente pas de problèmes technologiques particuliers du fait de l'absence de pièces en mouvement ou de composants actifs au contact du fluide cryogénique. La mesure de différence de pression peut être déportée en zone à température ambiante, cette facilité se fait au détriment de la bande passante de l'ensemble. 


\section{A. MEMPONTEIL}

Les inconvénients de cette technique sont également bien connus puisqu'ils sont dus à la difficulté d'obtenir une précision satisfaisante sur une grande dynamique avec un même organe de mesure. Dans notre cas, la dynamique de 100 sur le débit conduit à une gamme de différence de pression de 10000 à mesurer sur un seul dispositif déprimogène ce qui n'est pas envisageable du fait de la perte de charge induite à fort débit. La solution des dispositifs déprimogènes montés en parallèle et isolés par des vannes n'a pas été retenue car très onéreuse sur des circuits cryogéniques en double enceinte et d'autre part difficile à mettre en œuvre pendant les rafales où il pourrait se présenter des cas nécessitant un changement de gamme.

Le débitmètre à turbine semblait dès l'origine un candidat incontournable pour équiper l'installation. C'était la seule technique de mesure pour laquelle existait du matériel disponible explicitement spécifié pour mesurer des débits d'hydrogène liquide.

Le débitmètre à turbine possède entre autres avantages de pouvoir couvrir une plage de débit importante.

La mécanique de la turbine présente cependant quelques problèmes en raison de la faible viscosité de l'hydrogène qui impose l'utilisation d'un montage sur roulements à billes auto-lubrifiés, ce montage a été identifié comme un point faible potentiel de l'installation au cours des transitoires de mise en froid.

Enfin le débitmètre massique Coriolis par son principe, sa dynamique et sa robustesse mécanique nous est apparu très intéressant pour cette application.

Le coriolis fonctionne indifféremment en fluide incompressible ou non ; par principe il mesure le débit massique qui est la grandeur que nous souhaitons mesurer indépendamment de toute conversion basée sur des tables et tributaire de la précision d'autres mesures (pression, température).

Un inconvénient cependant pour cette technologie est que sur le marché il n'existe pas de matériel explicitement prévu pour fonctionner à $23 \mathrm{~K}$, et compte tenu de la complexité de cet instrument il n'était pas question que nous puissions en développer ou en modifier un.

Après analyse des possibilités nous avons opté pour l'utilisation de deux débitmètres en série par souci d'avoir une redondance de la mesure de débit même si celle-ci ne porte que sur une partie de la gamme des débits prévus. Le choix s'est porté sur un débitmètre massique coriolis et un débitmètre à turbine.

Les quantités mesurées sont dans un cas le débit massique dans l'autre le débit volumique.

Cette dualité augmente l'intérêt de la redondance car la densité du fluide présentant des variations d'un facteur deux, les deux mesures n'évoluent donc pas systématiquement de façon parallèle et des recoupements peuvent être faits pour diagnostiquer les éventuels dysfonctionnements.

\section{Débitmètre massique Coriolis}

Après consultation des différents fabriquants de débitmètres massiques coriolis, aucun matériel ne répondait à nos spécifications sur le point du fonctionnement à $23 \mathrm{~K}$.

Le matériel présentant les spécifications les plus proches était le DS025S de Rosemount Micro Motion. Un capteur similaire mais de gamme différente a déjà été testé sur de l'hydrogène liquide avec des résultats ne permettant pas de tirer de conclusions définitives sur son fonctionnement en hydrogène liquide.

Deux points d'interrogation demeurent sur le fonctionnement de ce matériel à très basse température.

Quel est le paramètre ayant amené le constructeur à fixer la limite inférieure de température de cet appareil à $33 \mathrm{~K}$ ?

En l'absence de cette information que nous n'avons pu obtenir, il ne nous est pas possible d'estimer la marge d'extrapolation tolérable de cette limite.

La deuxième interrogation porte sur les conséquences de la solidification de l'argon dans lequel baignent les parties mobiles du débitmètre lorsque celui-ci passe à une température inférieure à $84 \mathrm{~K}$. On peut supposer que le solide formé se dépose sur les parties mobiles de l'appareil dans lesquelles circule le fluide et qui sont les plus rapides à descendre en température : ce dépôt est-il en mesure de perturber le fonctionnement de l'appareil ?

Une réponse à cette interrogation serait de remplir l'enceinte de l'appareil d'hélium plutôt que d'argon pour les utilisations à très basse température.

Malgré ces interrogations nous avons installé un DS025S muni d'une électronique RFT 9712 sur le circuit d'essais à l'amont de la vanne d'entrée dans la section d'essais où l'écoulement est monophasique.

L'appareil a fait l'objet d'une vente conditionnelle de la part de la société Serve instrumentation qui distribue les matériels Rosemount. La condition d'achat était soumise au fonctionnement correct du capteur sur notre installation.

De fait le fonctionnement s'est avéré tout à fait satisfaisant.

Après mise en froid du capteur, le zéro de l'électronique est ajusté par la procédure prévue par le constructeur lorsque tout débit est annulé dans le débitmètre. La dérive de zéro entre la température ambiante et $25 \mathrm{~K}$ est d'environ $1,5 \mathrm{~g} / \mathrm{s}$ pour une pleine échelle de $200 \mathrm{~g} / \mathrm{s}$.

\section{Débitmètre à turbine}

Dans un premier temps nous avons fait appel à un matériel disponible spécifié pour fonctionner dans l'hydrogène liquide.

Ce matériel a été étalonné avec de l'air à 70 et 35 bar qui représente un compromis de similitude en Reynolds et densité. L'étalonnage a mis en évidence une dynamique de 5 pour une précision de $1 \%$ avec une loi linéaire.

Lors de la première mise en froid ce matériel a été détruit par une survitesse suivie d'une inversion de débit, nous l'avons donc retiré de l'installation.

En l'absence de matériel disponible correspondant à la fois aux spécifications du point de vue des performances et présentant des garanties de robustesse suffisantes, nous avons décidé de concevoir une turbine en privilégiant la robustesse mécanique de l'ensemble.

La conception de la turbine a été guidée par deux contraintes :

- Loger les roulements dont les dimensions et le positionnement sont imposés par la contrainte de tenue mécanique.

- Limiter la vitesse de rotation maximum à une valeur compatible avec une durée de vie acceptable des roulements tout en ayant à bas débit une vitesse de fluide suffisante pour ne pas limiter la dynamique. 
La turbine résultante pour un débit de $200 \mathrm{~g} / \mathrm{s}$ soit $3 \mathrm{l} / \mathrm{s}$ est représentée sur la figure 2.

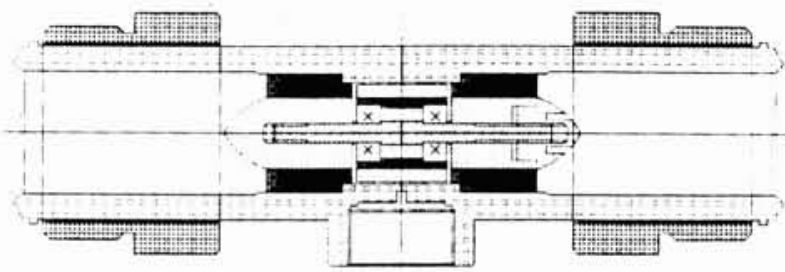

2. Turbine du débitmètre.

Les contraintes mentionnées plus haut conduisent à une hauteur de pales réduite à $2,5 \mathrm{~mm}$ un diamètre à la base de $11 \mathrm{~mm}$.

Cette géométrie est limitante pour la bande passante car elle implique une inertie importante. Elle a par contre l'avantage de faciliter l'usinage de la turbine en évitant le recours au gauchissement des pales.

La détection des pales est assurée par une bobine magnétique qui génère une résistance à la rotation, il serait préférable d'utiliser un détecteur à courant de Foucault afin d'augmenter la dynamique vers les faibles débits, malheureusement nous ne disposons pas actuellement de ce matériel.

L'étude mécanique et la réalisation du débitmètre ont été réalisées par la société SNLS.

\section{Validation des mesures}

Le débitmètre Coriolis est un matériel standard muni de son certificat d'étalonnage réalisé dans des conditions classiques (liquide à température ambiante). Pour son utilisation en hydrogène liquide il aurait été souhaitable de le faire tester avec ce fluide. Ce test était évidement difficile à réaliser.

Pour le débitmètre à turbine il aurait été souhaitable de faire un étalonnage en air sous pression de façon à analyser le comportement de la turbine. Pour des raisons de programme d'essais, cela n'a pas été possible. Nous avons donc utilisé l'installation d'essais elle-même et les moyens de calcul qui lui sont associés pour réaliser des essais de validation des mesures de débits.

\subsection{Comparaison Coriolis calcul en LH2}

Nous avons dans un premier temps utilisé le code monodimensionnel diphasique à deux fluides SIDHONY pour valider les débits mesurés avec le coriolis.

La comparaison calcul-mesure est faite sur le couple mesuré puis calculé (débit-perte de charge dans une tuyère).

La première section d'essais utilisée sur l'installation est une tuyère dont la géométrie et la rugosité sont connues avec précision.
L'entonnement de la tuyère est profilé sur une longueur égale à 13 fois le petit diamètre de façon à limiter les effets bidimensionnels, le rapport des sections vaut 5 et la longueur du col de la tuyère est de 70 L/D.

C'est cette tuyère qui est utilisé pour la validation des mesures de débit.

Lorsque l'écoulement dans la tuyère est monophasique, la perte de charge dans l'entonnement de la tuyère est due à l'accélération, aux frottements dans l'entonnement, aux effets bidimensionnels dans l'entonnement.

Le calcul de perte de charge par le code de calcul suffisamment maillé prend en compte les différents facteurs avec une rigueur différente.

L'accélération est calculée avec exactitude.

Le calcul de frottements est tributaire de la validité du coefficient de frottement. Celui-ci est ajusté sur l'ensemble des essais monophasiques afin que la ligne de pression calculée dans la section droite de la tuyère suive la ligne mesurée.

Le terme venant d'éventuels effets bidimensionnels ne peut être estimé avec rigueur, la tuyère n'ayant pas été étalonnée. On peut cependant estimer qu'il est négligeable devant les deux autres compte tenu de la géométrie de la tuyère.

Sur la figure 4 sont représentées un certain nombre de comparaisons mesure-calcul.

On constate que l'écart est d'environ $1 \%$, le calcul a tendance à sous-estimer le débit par rapport à la mesure.

La majorité de ces comparaisons est réalisée sur plage de débit réduite. Cela tient à ce que la comparaison du calcul en simple phase et de la mesure nécessite un sous refroidissement important du fluide dans la tuyère. Ceci est rarement le cas à fort débit où la perte de pression due à l'accélération dans la tuyère est importante.

Il faut noter que l'écart entre le calcul et la mesure est aussi tributaire de la mesure de température à l'entrée de la section d'essais et dans une moindre mesure de la pression, ces deux paramètres étant utilisés par le code pour le calcul de densité. Pour le point de la figure 3 pour lequel l'écart est le plus important, une variation de la température de $0,1 \mathrm{~K}$ entraîne un écart sur le débit calculé de $1,5 \%$.

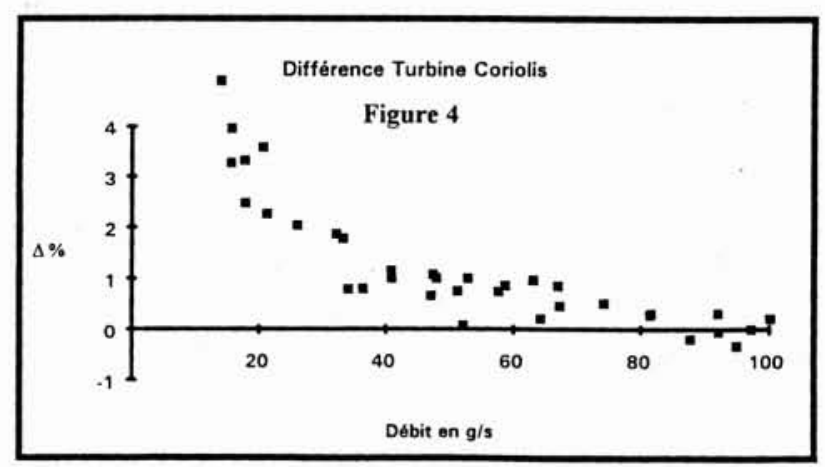

3. Ecart calcul-mesure Coriolis.

\subsection{Comparaison Coriolis turbine en LH2}

La figure 4 donne l'écart de mesure exprimé en débit massique entre le coriolis et la turbine lorsque les deux 
appareils ont été opérationnels. Le débit maximum est limité lors de ces tests par la section d'essais montée sur l'installation.

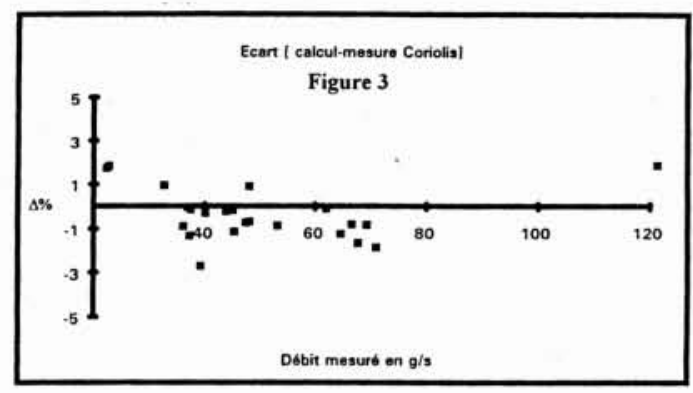

4. Ecart de mesure entre le coriolis et la turbine.

Les débits donnés par la turbine sont calculés à partir de la fréquence mesurée multipliée par le coefficient fréquence/débit théorique. Celui-ci est obtenu par le calcul de la section de passage du fluide multiplié par le pas de la turbine et divisé par le nombre de pales.

Les mesures sont dans une fourchette de $1 \%$ entre $40 \mathrm{~g} / \mathrm{s}$ et le maximum de débit atteint. La dynamique peut sembler ainsi restreinte mais il faut considérer que la turbine est capable de débits deux fois plus importants pour lesquels nous avons vérifié sa tenue mécanique à température ambiante mais pas sa précision.

Comme pour la comparaison mesure coriolis-calcul, l'estimation du débit masse vu par la turbine dépend de l'estimation de la densité du fluide qui est tributaire de la mesure de température.

\subsection{Comparaison Coriolis-turbine sur hydrogène gazeux}

Afin de tester le débitmètre massique à faible débit après les cyclages thermiques, nous avons utilisé une ligne d'in- jection d'hydrogène gaz, placée en série avec la section d'essais. Cette ligne est munie d'un débitmètre massique étalonné sur une gamme de 0 à $20 \mathrm{~g} / \mathrm{s}$ qui sert de référence.

Ces essais sont à température ambiante et limités en débit à $5 \mathrm{~g} / \mathrm{s}$ par le circuit.

Les deux débitmètres donnent dans ces conditions de fonctionnement un débit identique à $0,02 \mathrm{~g} / \mathrm{s}$ près, après une vingtaine de cyclages thermiques.

\section{Conclusion}

L'instrumentation de débitmétrie dont nous disposons sur le banc Mhynos donne actuellement satisfaction sur un certain nombre de points.

Les écarts présentés entre les mesures et le calcul marquent la cohérence entre les performances de la mesure de débit et, d'une part, le niveau d'approximation du calcul lorsque les modèles diphasiques qui doivent être développés ne sont pas en jeu, et d'autre part les autres mesures telles que la température et la pression qui permettent de caractériser le fluide.

Le fonctionnement du coriolis est très satisfaisant, sa dynamique est particulièrement intéressante pour une installation telle que Mhynos.

La validité des mesures à faible débit et à température cryogénique reste cependant à confirmer.

La turbine reste à améliorer sur le plan de la dynamique, ceci est en cours par une diminution de l'entrefer entre la turbine et le corps, l'adoption d'un détecteur à courant de Foucault permettrait sans doute de faire un pas significatif.

Un nouveau débitmètre est en cours de réalisation pour les faibles débits de 0 à $50 \mathrm{~g} / \mathrm{s}$.

\section{Références}

RANDAll F., Barron. Cryogenic systems: Oxford University Press 1985.

Perroud P., Rebière J. Convection forcée dans l'hydrogène liquide: Rapport CEA-R 24991965. 